\title{
Análise temporal dos planos de saúde médicos e odontológicos do Brasil
}

\author{
Temporal analysis of medical and dental health plans in Brazil \\ Análisis temporal de planes de salud médica y dental en Brasil
}

Recebido: 07/05/2021 | Revisado: 16/05/2021 | Aceito: 25/05/2021 | Publicado: 09/06/2021

\author{
Suzely Adas Saliba Moimaz \\ ORCID: https://orcid.org/0000-0002-4949-529X \\ Universidade Estadual Paulista, Brasil \\ E-mail: suzely.moimaz@unesp.br \\ Julio Martinez Alves Oliveira \\ ORCID: https://orcid.org/0000-0002-3173-9444 \\ Universidade Estadual Paulista, Brasil \\ E-mail: juliooliveira1994@hotmail.com \\ Giovani Hugo Rocha Botan \\ ORCID: https://orcid.org/0000-0001-9526-0611 \\ Universidade Estadual Paulista, Brasil \\ E-mail: giwbottan@gmail.com \\ Clea Adas Saliba Garbin \\ ORCID: https://orcid.org/0000-0001-5069-8812 \\ Universidade Estadual Paulista, Brasil \\ E-mail: clea.saliba-garbin@unesp.br \\ Tânia Adas Saliba \\ ORCID: https://orcid.org/0000-0003-1327-2913 \\ Universidade Estadual Paulista, Brasil \\ E-mail: tania.saliba@unesp.br
}

\begin{abstract}
Resumo
Os planos de saúde médicos e odontológicos apresentam uma expansão significativa no Brasil, logo torna-se necessário analisar as características desse tipo de prestação de serviço, como parte de um modelo de atenção à saúde. O objetivo deste estudo foi realizar uma análise descritiva das operadoras de planos de saúde médicos e odontológicos do Brasil e as características de seus beneficiários no período de 2009 a 2019. Trata-se de uma pesquisa realizada por meio de uma análise descritiva dos dados fornecidos pela plataforma de registro online da ANS. Foram analisadas as seguintes variáveis, no período de 2009 a 2019: número de operadoras e modalidades, faixa etária, tipo de plano e sexo dos beneficiários. Em 2009, havia 1498 operadoras de planos de saúde e em 2019, notaram-se 1022. Quanto aos beneficiários, havia 55 milhões de beneficiários em 2009 e em 2019 foram verificados 72 milhões, com predomínio do sexo feminino 53\%, e faixa etária entre 34 a 38 anos e modalidade coletiva empresarial, para ambas coberturas assistenciais, médica e odontológica. Conclui-se que houve crescimento expressivo no número de beneficiários dos planos de saúde, entretanto ocorreu um decréscimo na quantidade de operadoras de 2009 a 2019.
\end{abstract}

Palavras-chave: Saúde suplementar; Serviços de saúde; Planos de pré-pagamento em saúde.

\begin{abstract}
Medical and dental health plans present a significant expansion in Brazil, so it becomes necessary to analyze the characteristics of this type of serviceprovision, as part of a health care model. The objective of this study was to carry out a descriptive analysis of the medical and dental health plan operators in Brazil and the characteristics of their beneficiaries in the period from 2009 to 2019. This is a research conducted through a descriptive analysis of data provided by ANS online registration platform. The following variables were analyzed from 2009 to 2019: number of operators and modalities, age group, type of plan and gender of beneficiaries. In 2009 there were 1498 health insurance operators and in 2019, there were 1022. As for beneficiaries, there were 55 million beneficiaries in 2009 and in 201972 million were verified, with a predominance of 53\% females aged 34 to 38 years and collective corporate mode, for both medical and dental care coverage. It was concluded that there was a significant growth in the number of beneficiaries of health plans, however there was a decrease in the number of operators from 2009 to 2019.
\end{abstract}

Keywords: Supplemental health; Health services; Prepaid health plans.

\section{Resumen}

Los planes de salud médica y dental se están expandiendo significativamente en Brasil, por lo que se hace necesario analizar las características de este tipo de prestación de servicios, como parte de un modelo de atención de salud. 
El objetivo de este estudio fue realizar un análisis descriptivo de los operadores de planes de salud médica y dental en Brasil y las características de sus beneficiarios en el período de 2009 a 2019. Se trata de una investigación realizada a través de un análisis descriptivo de los datos proporcionados. por la plataforma de registro en línea ANS. Se analizaron las siguientes variables, en el período de 2009 a 2019: número de operadores y modalidades, grupo de edad, tipo de plan y sexo de los beneficiarios. En 2009 había 1498 operadores de planes de salud y en 2019 se registraron 1022. En cuanto a los beneficiarios, en 2009 había 55 millones de beneficiarios y en 2019 se verificaron 72 millones, con predominio del sexo femenino 53\%, y grupo de edad entre 34 y 38 años y modalidad de negocio colectivo, tanto para cobertura sanitaria, médica y odontológica. Se concluye que hubo un crecimiento significativo en el número de beneficiarios de los planes de salud, sin embargo hubo una disminución en el número de operadores de 2009 a 2019.

Palabras clave: Salud suplementaria; Servicios de salud; Planes de salud prepagos.

\section{Introdução}

Houve um crescimento significativo no número de indivíduos com planos privados de saúde nos últimos anos, atingindo cerca de 72 milhões de beneficiários (Agência Nacional de Saúde Suplementar, 2019).

Além da expansão, o sistema privado de planos e seguros de saúde experimentou grande diversificação nas modalidades empresariais, entretanto até meados de 1998 não estiveram submetidas à regulação pública. Apenas em 1998 foi aprovada legislação regulatória (Lei no 9.656, de agosto de 1998) (Brasil, 1998), que estabeleceu regras para os contratos e coberturas dos planos de saúde, e em 2000 foi criada a Agência Nacional de Saúde Suplementar, responsável pelo controle desse setor (Agência Nacional de Saúde Suplementar, 2019).

A Agência Nacional de Saúde Suplementar (ANS) tem finalidade institucional de promover a defesa do interesse público na assistência suplementar à saúde, regulando as operadoras setoriais, inclusive quanto às suas relações com prestadores $\mathrm{e}$ consumidores, contribuindo para o desenvolvimento das ações de saúde no país. Todos os procedimentos de normatização, fiscalização e controle das operadoras de planos privados de assistência à saúde são de competência da ANS (Agência Nacional de Saúde Suplementar, 2019).

A ANS possui uma plataforma de registros online disponíveis em sua página da web. Os estudos destes dados, permitem investigar a expansão e a distribuição dos beneficiários de planos de saúde no Brasil. Entretanto, a obtenção destas informações nem sempre são facilmente encontradas, impactando o acesso de gestores de saúde a estes dados. Neste sentido, os estudos dos planos de saúde, das operadoras e dos usuários destes serviços são de extrema importância por gerar informações aos gestores e às autoridades de saúde, sendo eles responsáveis por criar resoluções, instruções normativas e leis que buscam melhorar as condições dos serviços prestados por este segmento de saúde no país. A publicação de resoluções normativas pela ANS, pode causar impactos no cenário das operadoras de planos de saúde no Brasil. Em 2010, foi publicada uma destas resoluções, e que levou a mudanças nos últimos anos no setor (Ziroldo, Gimenes, \& Castelo Jr, 2013).

A chamada Saúde Suplementar adquire diversos formatos na prestação da assistência, podendo ser exclusivamente médico, médico/odontológico e exclusivamente odontológico. As operadoras de planos de saúde são classificadas, conforme seu estatuto jurídico, nas seguintes modalidades: Autogestão, Cooperativa Médica, Cooperativa Odontológica, Filantropia, Administradora, Seguradora especializada, Medicina de grupo e Odontologia de grupo (Agência Nacional de Saúde Suplementar, 2019).

Denomina-se "Autogestão" a empresa que opera planos de assistência à saúde destinados, exclusivamente, a empregados ativos, aposentados, pensionistas ou ex empregados, de uma ou mais empresas ou, ainda, a participantes e dependentes de associações de pessoas físicas ou jurídicas, fundações, sindicatos, entidades de classes profissionais ou assemelhados e seus dependentes. A modalidade "cooperativa" corresponde a uma sociedade sem fim lucrativo, conforme o disposto na Lei n 5.764 , de 16 de dezembro de 1971. Quando denominado "filantropia" é uma entidade sem fins lucrativos que opera planos privados de assistência à saúde, sendo certificada como entidade filantrópica junto ao Conselho Nacional de Assistência Social (CNAS), e 
declarada de utilidade pública pelo Ministério da Justiça e pelos órgãos dos governos estaduais e municipais. Modalidade "administradora" é a empresa que apenas administra planos de assistência à saúde, que são financiados por outra operadora. Uma administradora não assume o risco decorrente da operação desses planos e não possui rede própria, credenciada ou referenciada de serviços médico-hospitalares ou odontológicos. A "seguradora autorizada" sociedade seguradora autorizada a operar planos de saúde, desde que esteja constituída como seguradora especializada nesse tipo de seguro, devendo seu estatuto social vedar a atuação em quaisquer outros ramos ou modalidade. Já a medicina e odontologia de grupo são as demais empresas ou entidades que operam planos de saúde (Agência Nacional de Saúde Suplementar, 2019).

Com a caracterização da Assistência Odontológica Suplementar surgiram as empresas odontológicas, cujo principal objetivo parece ser obter o maior lucro possível num mercado de serviços no qual há muitos cirurgiões-dentistas e muitas pessoas sem acesso ao atendimento. O profissional vê esses planos de saúde como uma oportunidade de aumentar sua clientela e seu faturamento, acarretando talvez, numa busca frenética por convênios que nem sempre proporcionam condições ideais para a prática da odontologia liberal (Mendes, 2005).

A presença de demanda no caso de planos odontológicos é potencializada pelo excesso de oferta no mercado de cirurgiões-dentistas. De acordo com Conselho Federal de Odontologia (CFO), houve aumento no número de cirurgiões dentistas no decorrer dos anos no Brasil, chegando a 323.000 profissionais registrados no conselho em 2019. A OMS recomenda 1 cirurgião dentista para cada 1500 habitantes, entretanto o estado de São Paulo apresenta a maior concentração de profissionais, tendo como relação a 1 cirurgião dentista para cada 476 habitantes. A disparidade na distribuição de profissionais no território nacional, com concentração nos grandes centros têm gerado um aumento na concorrência entre os cirurgiões dentistas em determinadas regiões (Zanetti, 1999). Embora a Agência Nacional de Saúde Suplementar (ANS) defina um rol de procedimentos de cobertura obrigatória, a instituição de protocolos e fluxos adotados e não regulados, acabam dificultando e fragmentando o cuidado, interferindo na qualidade da assistência (Malta, Cecílio, Merhy, Franco, Jorge, \& Costa, 2004). Tal realidade foi comprovada no segmento de planos médicos (Andrade, Freitas, Cherchiglia, \& Lana, 2009; Barros, Lopes, Souza, Tamaki, Teixeira, 2009; Cecílio, Aciole, Meneses, \& Iriart, 2005). Diante desse contexto, se faz importante o conhecimento do setor de saúde suplementar no Brasil, sua evolução numa serie temporal, visando fornecer subsídios para a informação pública e estabelecimentos de estratégias para prestação de serviços de saúde à população.

O objetivo deste estudo foi realizar uma análise descritiva das operadoras de planos de saúde médicos e odontológicos do Brasil e as características de seus beneficiários no período de 2009 a 2019.

\section{Metodologia}

Trata-se de um estudo descritivo, de caráter quantitativo, documental, e exploratório que foi realizado por meio de pesquisas de documentos, normas e portarias divulgadas nas bases de dados disponíveis na plataforma online da Agência Nacional de Saúde Suplementar, e que enfatizam os dados relacionados ás características dos beneficiários de planos de saúde médicos e odontológicos no Brasil e das operadoras destes planos. Nos métodos quantitativos, faz-se a coleta de dados quantitativos ou numéricos por meio do uso de medições de grandezas e obtém-se por meio da metrologia, números com suas respectivas unidades. (Pereira et al., 2018)

Inicialmente foi feito um treinamento à distância dos pesquisadores, com uma técnica da ANS através dos canais de atendimento da plataforma online da Agência Nacional de Saúde Suplementar (ANS). Nesse treinamento foram obtidas informações sobre a utilização das ferramentas de pesquisas fornecidos pela plataforma online. A plataforma de registros online TABNET, responsável pelo armazenamento de dados de saúde do DATASUS (Departamento de informática do SUS), foi consultada para a coleta dos dados sobre as operadoras de planos médicos, odontológicos e as características de seus beneficiários, no período de março de 2009 a janeiro de 2019. 
As variáveis foram consultadas em uma série temporal de 2009 a 2019. Este período foi selecionado em função da publicação da resolução normativa RN n. 211, de 11 de Janeiro de 2010, além da disponibilidade de dados da plataforma da Agência Nacional de Saúde Suplementar, bem como pelo interesse em investigar as mudanças ocorridas neste período, que conheceu um crescimento expressivo do setor no número de beneficiários em planos privados de assistência médica e odontológica,com destaque para o segmento odontológico. Em relação as operadoras de planos de saúde, as variáveis estudadas foram o número de operadoras de planos de saúde médico e odontológico no período de 2009 a 2019, e o número de operadoras segundo a modalidade no ano de 2019. Sobre os beneficiários de planos de saúde, foi investigado o número de beneficiários de acordo com a região do Brasil em 2019, o número de beneficiários de acordo com o tipo de cobertura assistencial no período de 2009 a 2019, o número de beneficiários de planos de saúde de acordo com a cobertura assistencial e tipo de contratação no ano de 2019, o número de beneficiários de planos de saúde de assistência exclusivamente odontológica segundo o sexo e faixa etária em 2019, e o número de beneficiários de planos de saúde de assistência médica com ou sem odontologia segundo o sexo e faixa etária no ano de 2019.

$\mathrm{Na}$ classificação das Operadoras as seguintes modalidades foram consideradas: Autogestão, Cooperativa Médica, Filantropia, Administradora, Seguradora especializada em Saúde, Cooperativa Odontológica, Odontologia de Grupo, de acordo com a classificação estabelecida pela ANS (Agência Nacional de Saúde Suplementar, 2019). De acordo com análise descritiva dos dados, foram apresentadas tabelas e gráficos por meio do excel 2017.

\section{Resultados}

Houve redução contínua no número de operadoras nos últimos 10 anos, como mostra a Tabela 1.

Tabela 1. Número de operadoras de planos de saúde, segundo o ano. Brasil, 2009-2019.

\begin{tabular}{crrr}
\hline Ano & $\begin{array}{c}\text { Médico-hospitalares } \\
\text { com ou sem Odontologia }\end{array}$ & $\begin{array}{c}\text { Exclusivamente } \\
\text { Odontológicas }\end{array}$ & Total \\
\hline 2009 & 1.095 & 403 & 1.498 \\
2010 & 1.045 & 374 & 1.419 \\
2011 & 1.015 & 369 & 1.384 \\
2012 & 962 & 359 & 1.321 \\
2013 & 920 & 344 & 1.264 \\
2014 & 874 & 343 & 1.217 \\
2015 & 828 & 327 & 1.155 \\
2016 & 790 & 305 & 1.095 \\
2017 & 766 & 291 & 1.057 \\
2018 & 749 & 289 & 1.038 \\
2019 & 737 & 285 & $1.022^{*}$ \\
\hline
\end{tabular}

Nota*: No ano de 2019 foram analisadas as operadoras até Junho. Fonte: Base de dados ANS (2019).

Em 2019 observou-se um total de 737 operadoras médico-hospitalares e 285 operadoras de planos exclusivamente odontológicos, totalizando 1022 operadoras, classificadas nas seguintes modalidades: Autogestão (156), Cooperativa Médica (286), Filantropia (39), Medicina de Grupo (247), Seguradora Especializada em Saúde (9), Cooperativa Odontologia (105), Odontologia de Grupo (180), como demonstrado na Tabela 2. 
Tabela 2. Número de operadoras de planos de saúde, segundo a modalidade. Brasil, 2019.

\begin{tabular}{lc}
\hline Modalidade & Operadoras com Beneficiários \\
\hline Autogestão & 156 \\
Filantropia & 39 \\
Seguradora Especializada em Saúde & 9 \\
Cooperativa Médica & 286 \\
Cooperativa Odontológica & 105 \\
Medicina de Grupo & 247 \\
Odontologia de Grupo & 180 \\
Total & 1.022 \\
\hline
\end{tabular}

Fonte: Base de dados ANS (2019).

Os beneficiários estão, em sua maioria, na região Sudeste $(60,2 \%)$ seguido pela região Nordeste $(15,8 \%)$, Sul $(13,2 \%)$, Centro-Oeste $(6,6 \%)$, Norte $(4 \%)$ e não identificado $(0,1)$. Do total de 72 milhões de beneficiários, 34,4\% correspondem a beneficiários de planos exclusivamente odontológicos e 65,6\% possuem planos Assistenciais médicos, alguns deles com assistência odontológica. A tabela 3 apresenta o número de beneficiários por região do Brasil e o tipo de cobertura.

Tabela 3. Número absoluto de beneficiários de planos de saúde, segundo as regiões. Brasil, 2019.

\begin{tabular}{lrr}
\hline Região & Assistência Médica & $\begin{array}{c}\text { Exclusivamente } \\
\text { Odontológica }\end{array}$ \\
\hline Centro - Oeste & 3.205 .754 & 1.557 .276 \\
Nordeste & 6.605 .675 & 4.819 .853 \\
Norte & 1.732 .927 & 1.140 .931 \\
Sudeste & 28.800 .145 & 14.643 .478 \\
Sul & 6.956 .059 & 2.588 .360 \\
Não Identificado & 32.351 & 49.789 \\
\hline
\end{tabular}

Fonte: Base de dados ANS (2019).

Analisando os últimos 10 anos, nota-se um crescimento no número de beneficiários de Planos de Saúde Médicos com ou sem assistência Odontológica de 2009 à 2014. A partir de 2014, notou-se redução continua até o ano de 2019. Em contrapartida o número de beneficiários de Planos exclusivamente odontológicos apresentou um crescimento expressivo e anual de 2009 até 2019 chegando a 24 milhões de beneficiários, como mostra a Tabela 4. 
Tabela 4. Número de beneficiários de planos privados de saúde, de acordo com o tipo de cobertura assistencial. Brasil, 2009-2019.

\begin{tabular}{ccc}
\hline Ano & $\begin{array}{c}\text { Beneficiários em planos } \\
\text { privados de assistência } \\
\text { médica com ou sem } \\
\text { odontologia }\end{array}$ & $\begin{array}{c}\text { Beneficiários em planos } \\
\text { privados exclusivamente } \\
\text { odontológicos }\end{array}$ \\
\hline 2009 & 42.561 .398 & 13.253 .744 \\
2010 & 44.937 .350 & 14.514 .074 \\
2011 & 46.025 .814 & 16.669 .935 \\
2012 & 47.846 .092 & 18.538 .837 \\
2013 & 49.491 .826 & 19.561 .930 \\
2015 & 50.482 .636 & 20.086 .828 \\
2016 & 49.242 .995 & 20.845 .243 \\
2017 & 47.638 .144 & 21.202 .256 \\
2018 & 47.124 .593 & 22.392 .230 \\
2019 & 47.213 .519 & 24.260 .858 \\
\hline
\end{tabular}

Fonte: Base de dados ANS (2019).

As contratações dos planos podem ocorrer de forma individual, familiar ou coletiva. Os planos de saúde coletivos são a maioria, representando $81 \%$ do total de contratações (Tabela 5).

Tabela 5. Número de beneficiários de planos de saúde, de acordo com a cobertura assistencial e tipo de contratação. Brasil - 2019.

\begin{tabular}{cr}
\hline Cobertura assistencial e tipo de contratação do plano & Número de \\
& Beneficiários \\
\hline Assistência médica com ou sem odontologia & \\
Individual ou Familiar & 9.036 .063 \\
Coletivo Empresarial & 31.632 .092 \\
Coletivo por adesão & 6.358 .948 \\
Coletivo não identificado & 1.103 \\
Não Informado & 97.644 \\
\hline Subtotal & $\mathbf{4 7 . 1 2 5 . 8 5 0}$ \\
\hline Exclusivamente odontológicos & 4.377 .703 \\
Individual ou Familiar & 18.139 .013 \\
Coletivo Empresarial & 2.238 .922 \\
Coletivo por adesão & 2.458 \\
Coletivo não identificado & 13.751 \\
Não Informado & 24.771 .847 \\
\hline Subtotal & \\
\hline
\end{tabular}

Fonte: Base de dados ANS (2019).

A faixa etária que concentra a maior parte dos beneficiários dos planos exclusivamente odontológicos é entre os 24 e 43 anos e abaixo dos 18 anos, representando 65,2\% do total de beneficiários (Tabela 6). 
Tabela 6. Número de beneficiários de planos de saúde de assistência exclusivamente odontológica, segundo sexo e faixa etária. Brasil, 2019.

\begin{tabular}{lrrr}
\hline Faixa etária & Masculino & Feminino & Total \\
\hline 00 a 18 anos & 2.472 .433 & 2.382 .424 & 4.854 .857 \\
19 a 23 anos & 953.067 & 999.728 & 1.952 .795 \\
24 a 28 anos & 1.189 .921 & 1.295 .150 & 2.485 .071 \\
29 a 33 anos & 1.467 .063 & 1.566 .971 & 3.034 .034 \\
34 a 38 anos & 1.547 .133 & 1.615 .290 & 3.162 .423 \\
39 a 43 anos & 1.291 .747 & 1.341 .654 & 2.633 .401 \\
44 a 48 anos & 968.071 & 1.024 .269 & 1.992 .340 \\
49 a 53 anos & 762.792 & 808.352 & 1.571 .144 \\
54 a 58 anos & 575.236 & 620.154 & 1.195 .390 \\
59 anos ou mais & 876.637 & 1.013 .363 & 1.890 .000 \\
Inconsistente & 187 & 205 & 392 \\
Total & 12.104 .287 & 12.667 .560 & 24.771 .847 \\
\hline
\end{tabular}

Fonte: Base de dados ANS (2019).

Já nos planos de assistência Médica com ou sem odontologia, a faixa etária mais concentrada é dos 00 aos 18 anos, seguida por "59 anos ou mais", representados na Tabela 7.

Tabela 7. Número de beneficiários de planos de saúde de assistência médica com ou sem odontologia, segundo sexo e faixa etária. Brasil, 2019.

\begin{tabular}{lrrr}
\hline Faixa etária & Masculino & Feminino & Total \\
\hline 00 a 18 anos & 5.747 .182 & 5.527 .279 & 11.274 .461 \\
19 a 23 anos & 1.476 .085 & 1.582 .831 & 3.058 .916 \\
24 a 28 anos & 1.670 .416 & 1.967 .226 & 3.637 .642 \\
29 a 33 anos & 2.083 .107 & 2.468 .613 & 4.551 .720 \\
34 a 38 anos & 2.283 .746 & 2.625 .157 & 4.908 .903 \\
39 a 43 anos & 1.964 .174 & 2.211 .769 & 4.175 .943 \\
44 a 48 anos & 1.520 .828 & 1.717 .988 & 3.238 .816 \\
49 a 53 anos & 1.296 .537 & 1.486 .132 & 2.782 .669 \\
59 anos ou mais & 2.844 .149 & 4.148 .536 & 6.992 .685 \\
Inconsistente & 279 & 253 & 532 \\
Total & 22.033 .203 & 25.092 .647 & 47.125 .850 \\
\hline
\end{tabular}

Fonte: Base de dados ANS (2019). 


\section{Discussão}

No Brasil, a saúde suplementar vem se destacando pela quantidade e também pela qualidade dos serviços prestados aos seus usuários. (Ziroldo, Gimenes, \& Castelo Jr, 2013). Estudos indicaram que usuários de planos de saúde privados fizeram maior uso de serviços de saúde comparados com indivíduos que não apresentam planos, e relataram melhores condições de saúde . (Manski, Moeller, \& Chen, 2014; Moraes, Moreira, \& Luiz, 2011; Porto, Ugá, \& Moreira, 2011; Teusner, Brennan, \& Spencer, 2015).

Nesta pesquisa sobre a saúde suplementar no Brasil, verificou-se que houve uma diminuição no número de operadoras e um aumento no número de beneficiários entre 2009 e 2019. Essa parcela da população pode acessar o sistema de saúde de forma duplicada, tanto pelo uso do serviço público quanto pelo uso por meio do plano privado (Garcia-Subirats, Vargas, Mogollón-Pérez, De Paepe, Silva, Unger, Borrell, \& Vázquez, 2014).

A Agência Nacional de Saúde Suplementar publicou uma resolução normativa ampliando o rol de procedimentos que devem ser cobertos pela Saúde Suplementar. Trata-se da normativa RN n. 211, que beneficia contratos assinados após a entrada em vigor da lei n. 9.656 (Ziroldo et al., 2013).

De acordo com as operadoras de planos de saúde do segmento da medicina de grupo, esta mudança pode causar impactos econômicos negativos em empresas de pequeno e médio porte, já que a ANS irá avaliar os custos para estas operadoras apenas um ano após a vigência desta normativa. (Ziroldo et al., 2013).

A dificuldade das empresas deste setor em se manterem ativas, está levando a um processo de fusão entre elas, caracterizando o cenário atual da Saúde Suplementar no Brasil. (Ziroldo et al., 2013). Nos últimos 10 anos, reformas no sistema de saúde suplementar em diversos países também foram destaque, como o caso dos Estados Unidos. A reforma aprovada no país norte-americano inclui medidas tendentes a aumentar a cobertura, desenvolver a promoção da saúde e a prevenção da doença, melhorar a acessibilidade, aumentar a eficiência, melhorar a qualidade, reforçar a regulação e reduzir os custos (Costa, 2013). No Brasil, apesar do número de beneficiários dos planos de saúde apresentar um crescimento significativo de 2009 a 2019 o mercado de planos privados é concentrado. Embora existam mais de mil empresas, a maioria dos beneficiários estão concentrados em poucas operadoras, o que pode justificar a constante redução do número de operadoras de planos de saúde.

De acordo com a ANS, 55 milhões de pessoas eram atendidas pela Saúde Suplementar em 2009 no país. Este número chegou em 72 milhões em 2019, um crescimento de aproximadamente 32\%. Do total vínculos aos planos de saúde, cerca de 47 milhões são de assistência médica com ou sem odontologia e 24 milhões de assistência exclusivamente odontológica, o que significa uma cobertura aproximada de $34 \%$ da população brasileira (IBGE aponta que 71,5\% da população brasileira depende do SUS, 2019) distribuída de maneira desigual entre as regiões do país, maior entre os residentes nas áreas urbanas e nos estados com maior renda e maior oferta de emprego formal e de serviços de saúde, situação encontrada nas regiões Sudeste Sul e Nordeste (Albuquerque, Piovesan, Santos, Martins, Fonseca, \& Sasson, 2008).

Considerando-se que a Saúde Suplementar é uma atividade com fins lucrativos, que implica no pagamento por parte de seus beneficiários pelos serviços prestados, há de se indagar os motivos pelos quais milhares de brasileiros estão aderindo a esse sistema, mesmo considerando o direito à saúde garantido na constituição brasileira. O Sistema Único de Saúde (SUS), criado pela lei 8080/90 deve garantir o acesso e cuidado integral à saúde de todo cidadão brasileiro (Brasil, 1990), contudo a falta de resolutividade, as filas de espera e dificuldade de atendimento podem estar relacionadas no aumento da Saúde Suplementar.

Em pesquisa realizada com usuários do SUS, verificou-se que a insatisfação quanto ao tempo de espera por um tratamento tem-se mostrada rotineira nos serviços de saúde (Moimaz, Marques, Saliba, Garbin, Zina, \& Saliba, 2010).

O expressivo crescimento da saúde suplementar nos planos exclusivamente odontológicos pode estar relacionado com a busca por atendimentos odontológicos especializados, embora existam os CEOs (Centro de Especialidade Odontológica), locais de referência para atendimento odontológico no SUS. 
Existem países em que a organização da interface entre a atenção primária e a secundária dos serviços odontológicos funciona de maneira organizada e integrada, para suprir a necessidade de atendimento de sua população (Lo, Lin, Wang, Wong, \& Schwarz, 2001; Morris, \& Burke, 2001; Nuttall, Steed, \& Donachie, 2002; Scott, Brodeur, Olivier, \& Benigeri, 2002). Porém, para que haja efetiva utilização dos serviços de saúde, não basta apenas uma maior disponibilidade e oferta, é necessário analisar determinados fatores, como a distribuição geográfica, a facilidade de acesso ao atendimento odontológico, as mudanças do perfil epidemiológico e as características da gestão do serviço. Torna-se um desafio planejar medidas de saúde pública que atendam aos grupos mais vulneráveis, pois o padrão de utilização dos serviços e as desigualdades nos padrões de doença estão diretamente relacionados à desigualdade social (Antunes, \& Narvai, 2010; Chaves, Cruz, Barros, \& Figueiredo, 2011).

O plano de assistência à saúde mais frequente foi o de contratação coletiva (empresarial ou individual). Entende-se como plano de saúde de contratação coletiva empresarial, aquele que oferece cobertura da atenção prestada à população delimitada e vinculada a pessoa jurídica. O vínculo referido poderáser de caráter empregatício, associativo ou sindical. O contrato poderá prever a inclusão dos dependentes legais da massa populacional vinculada e a adesão deverá ser automática na data da contratação do plano ou no ato da vinculaçãodo consumidor à empresa, associação ou sindicato, de modo a abranger a totalidade ou a maioria absoluta da massa populacional vinculada. Já os planos coletivos individuais são aqueles oferecidos no mercado para a livre adesão de consumidores, pessoas físicas, com ou sem seu grupo familiar. Caracteriza-se o plano como familiar quando facultada ao contratante, pessoa física, a inclusão de seus dependentes ou grupo familiar.

A maior frequência de planos coletivos empresariais, aliada a faixa etária de maior número de beneficiários que é a de 34 a 38 anos, provavelmente está relacionada à classe trabalhadora. A saúde da mulher foi incorporada às políticas nacionais de saúde nas primeiras décadas do século XX, com a ação dos movimentos feministas, e, desde então, evolui de maneira positiva (Barbosa, 2014). Ressalta-se que no sexo feminino, a faixa de maior frequência de beneficiários dos planos de assistência médica é a de 34 a 38 anos de idade, possivelmente em razão das necessidades de cuidados pré-natais, considerando que, atualmente as mulheres estão optando por engravidarem com mais idade, após terem garantido sua inserção no mercado de trabalho. Esse fato foi observado também na pesquisa realizada por Alves, Feitosa, Mendes, \& Caminha (2017).

$\mathrm{O}$ crescimento do mercado de planos de assistência médica que acompanhou o incremento da população brasileira. Grande parte dos beneficiários está na faixa etária de 20 a 49 anos e concentra-se nos municípios mais populosos e de maior industrialização do país, vinculados na sua maioria a planos coletivos, possivelmente devido à inserção no mercado de trabalho. Esses achados foram também relatados em outro estudo (Albuquerque et al., 2008). Observou- se, também, que a procura por planos de saúde de Assistência Médica é maior do que exclusivamente odontológicos, por beneficiários acima dos 59 anos, podendo estar relacionado com a maior necessidade de atenção e atendimento médico especializado da população idosa.

A saúde suplementar no Brasil apresenta um crescimento significativo, entretanto, a maior parte dos Brasileiros são dependentes do Sistema Único de Saúde. Muitos usuários de planos de saúde também utilizam o SUS. De acordo com o IBGE (Instituto Brasileiro de Geografia e Estatística ,2007) o Brasil possuía 184 milhões de habitantes em 2007, sendo 136,2 milhões de brasileiros dependentes exclusivamente do SUS. (Ziroldo, Gimenes, \& Castelo Jr, 2013). No ano de 2019, o país era composto por aproximadamente 210 milhões de habitantes de acordo com o IGBE, e 150 milhões utilizavam exclusivamente o SUS para tratamento, cerca de 71,5\% da população. (Instituto Brasileiro de Geografia e Estatística, 2020).

No ano de 2004 foi elaborada uma pesquisa para avaliar a satisfação de usuários de um posto de saúde do SUS. Dos entrevistados, 32,7\% relataram que iriam continuar a usar o serviço público de saúde pelo fato de não terem acesso um serviço de saúde privado. (Traverso-Yépez, \& Morais, 2004). Diante da dificuldade do cidadão ter acesso a serviços de saúde de alta complexidade no sistema público de saúde e do atual cenário de saúde no país, a adesão a um plano de saúde tornou-se necessidade (Almeida, 2009). As dificuldades do acesso aos serviços de saúde vão muito além do aspecto geográfico, e se relacionam, principalmente, à baixa oferta de serviços. Além disso, devem ser considerados os aspectos organizacionais; 
econômicos; sociais; culturais; religiosos; epidemiológicos e de comunicação com as equipes de saúde (Figueira, Silva, \& Silva, 2018; Oliveira, Gabriel, Poz, \& Dussault, 2017).

Em contrapartida, há também empecilhos na cobertura desses procedimentos, por parte dos planos de saúde, motivo de queixas e reclamações de seus beneficiários, gerando processos em órgãos de defesa de consumidor (Vieira Junior, \& Martins, 2015).A saúde suplementar deve ser monitorada e regulada constantemente, para garantia da qualidade dos serviços prestados aos beneficiários, bem como para conhecimento dos entraves existentes entre o direito do cidadão e as obrigações das empresas sejam resolvidos satisfatoriamente. É possível destacar que a avaliação deve fazer parte do processo de trabalho em saúde, com o intuito de promover mudanças em suas dimensões de produtividade, satisfação e realização pessoal dos profissionais e dos usuários, economia de meios e otimização de recursos, e assim, adequá-los à realidade (Yamamoto, Machado, \& Silva Junior, 2015).

\section{Conclusão}

Conclui-se que houve crescimento expressivo no número de beneficiários dos planos de saúde, entretanto ocorreu um decréscimo na quantidade de operadoras de 2009 a 2019. A maioria dos beneficiários concentram-se na região Sudeste, predominando o sexo feminino, e o tipo de contratação dos planos de saúde mais frequente é a coletiva empresarial. Este estudo limita-se a consulta dos bancos de dados disponíveis na plataforma online da Agência Nacional de Saúde Suplementar, e portanto foram analisados dados quantitativos, não sendo aprofundadas questões relacionadas a coberturas de planos e problemas operacionais, os quais podem ser investigados em estudos futuros por meio da aplicação de inquéritos entre usuários de planos de saúde. O acesso e a obtenção das informações mesmo estando disponíveis na plataforma online da ANS, nem sempre são facilmente encontradas. Foi preciso realizar um treinamento à distância dos pesquisadores, com uma técnica da ANS por meio dos canais de atendimento da plataforma online da Agência Nacional de Saúde Suplementar (ANS). Entretanto, esta pesquisa contribui para elucidar a evolução em uma série temporal da tendência de crescimento do número de beneficiários dos planos de saúde no brasil, podendo assim os gestores e legisladores de saúde promover a regulação de normas que buscam garantir os direitos e deveres dos usuários destes serviços e das operadoras prestadoras de serviços de saúde suplementar.

\section{Agradecimentos}

O presente trabalho foi realizado com apoio da Coordenação de Aperfeiçoamento de Pessoal de Nível Superior Brasil (CAPES) - Código de Financiamento 001.

\section{Referências}

Agência Nacional de Saúde Suplementar (2019). Quem somos. http://www.ans.gov.br/aans/quem-somos

Albuquerque, C., Piovesan, M. F., Santos, I. S., Martins, A. C. M., Fonseca, A. L., \& Sasson, D. (2008). A situação atual do mercado da saúde suplementar no Brasil e apontamentos para o futuro. Ciência \& Saúde Coletiva, 13(5), 1421-1430. https://doi.org/10.1590/S1413-81232008000500008

Almeida, A. (2009). Newsletter Abramge http://www.abramge.com.br/imagens/banco/file/Clippings/InvestNews\%20-\%2006_02.pdf

Alves, N. C. C., Feitosa, K. M. A., Mendes, M. E. S., \& Caminha, M. F. C. (2018). Complicações na gestação em mulheres com idade maior ou igual a 35 anos. Revista Gaúcha de Enfermagem, 38(4), e2017-0042. https://doi.org/10.1590/1983-1447.2017.04.2017-0042

Andrade, E. I. G., Freitas, R. M., Cherchiglia, M. L., \& Lana, F. C. F. (2009). Análise dos modelos assistenciais praticados pelas operadoras de planos de saúde em Minas Gerais. In R. C. Pereira, R. M. Silvestre (Orgs.), Regulação e modelos assistenciais em saúde suplementar: produção cientifica da Rede de Centros Colaboradores da ANS - 2006/2008 (pp. 87-118). Brasília: Organização Pan-Americana da Saúde.

Antunes, J. L. F., \& Narvai, P. C. (2010). Políticas de saúde bucal no Brasil e seu impacto sobre as desigualdades em saúde. Revista de Saúde Pública, 44(2), 360-365. https://doi.org/10.1590/S0034-89102010005000002

Barbosa, C. J. L. (2014). Saúde do homem na atenção primária: mudanças necessárias no modelo de atenção. Revista Saúde e Desenvolvimento, 6(3), 99-114. 
Barros, M. E. D., Lopes, G. C. F. R., Souza, M. R., Tamaki, E. M., \& Teixeira, L. S. (2009). Mercado de saúde suplementar na região Centro- Oeste. In R. C. Pereira, R. M. Silvestre (Orgs.), Regulação e modelos assistenciais em saúde suplementar: produção cientifica da Rede de Centros Colaboradores da ANS 2006/2008 (pp. 43-86). Brasília: Organização Pan-Americana da Saúde.

Brasil (1990). Lei nº 8.080, de 19 de setembro de 1990. Lei Orgânica da Saúde. Dispõe sobre as condições para a promoção, proteção e recuperação da saúde, a organização e o funcionamento dos serviços correspondentes e dá outras providências. http://www.planalto.gov.br/ccivil_03/leis/18080.htm

Brasil (1998). Lei $\mathrm{n}^{\circ}$ 9.656, de 3 de junho de 1998. Dispõe sobre os planos e seguros privados de assistência à saúde. http://www.planalto.gov.br/ccivil_03/leis/19656.htm

Cecílio, L. C. O., Aciole, G. G., Meneses, C. S., \& Iriart, C. B. (2005). A microrregulação praticada pelas operadoras investigadas. In: Brasil. Duas faces da mesma moeda: microrregulação e modelos assistenciais na saúde suplementar (pp. 75-140). Rio de Janeiro: Ministério da Saúde.

Chaves, S. C. L., Cruz, D. N., Barros, S. G., \& Figueiredo, A. C. L. (2011). Avaliação da oferta e utilização de especialidades odontológicas em serviços públicos de atenção secundária na Bahia, Brasil. Cadernos de Saúde Pública, 27(1), 143-154. https://doi.org/10.1590/S0102-311X2011000100015

Costa, J. P. (2013). A reforma Obama e o sistema de saúde dos EUA. Arquivos de Medicina, 27(4), 158-167.

Figueira, M. C. S., Silva, W. P., \& Silva, E. M. (2018). Acesso aos serviços da Atenção Primária em Saúde: revisão integrativa da literatura. Revista Brasileira de Enfermagem, 71(3), 1178-1188. https://doi.org/10.1590/0034-7167-2017-0441

Garcia-Subirats, I., Vargas, I., Mogollón-Pérez, A. S., De Paepe, P., Silva, M. R., Unger, J. P., Borrell, C., \& Vázquez, M. L. (2014). Inequities in access to health care in different health systems: a study in municipalities of central Colombia and north-eastern Brazil. International Journal for Equity in Health, 13, 10. https://doi.org/10.1186/1475-9276-13-10

IBGE aponta que 71,5\% da população brasileira depende do SUS (2020). http://atarde.uol.com.br/saude/noticias/2137933-ibge-aponta-que-715-da-populacaobrasileira-depende-do-sus\#: :text=O\%20Instituto\%20Brasileiro\%20de\%20Geografia,havia\%20chegado\%20ainda\%20no\%20pa\%C3\%ADs

Instituto Brasileiro de Geografia e Estatística (2007). http://www.ibge.com.br/home/estatistica/populacao/contagem2007/contagem_final/tabela1_1.pdf

Lo, E. C., Lin, H. C., Wang, Z. J., Wong, M. C., \& Schwarz, E. (2001). Utilization of dental services in Southern China. Journal of dental research, 80(5), 14711474. https://doi.org/10.1177/00220345010800051701

Malta, D. C., Cecílio, L. C. O., Merhy, E. E., Franco, T. B., Jorge, A. O., \& Costa, M. A. (2004). Perspectivas da regulação na saúde suplementar diante dos modelos assistenciais. Ciência \& Saúde Coletiva, 9(2), 433-444. https://doi.org/10.1590/S1413-81232004000200019

Manski, R. J., Moeller, J. F., \& Chen, H. (2014). Dental care coverage and use: modeling limitations and opportunities. American Journal of Public Health, 104(2), e80-e87. https://doi.org/10.2105/AJPH.2013.301693

Mendes, H. J. (2005). A relação entre cirurgiões-dentistas e as operadoras de planos de saúde no município de Bauru-SP (Dissertação de Mestrado). Faculdade de Odontologia, Universidade de São Paulo, Bauru.

Moimaz, S. A. S., Marques, J. A. M., Saliba, O., Garbin, C. A. S., Zina, L. G., \& Saliba, N. A. (2010). Satisfação e percepção do usuário do SUS sobre o serviço público de saúde. Physis: Revista de Saúde Coletiva, 20(4), 1419-1440. https://doi.org/10.1590/S0103-73312010000400019

Moraes, J. R., Moreira, J. P. L., \& Luiz, R. R. (2011). Associação entre o estado de saúde autorreferido de adul - tos e a área de localização do domicílio: uma análise de regressão logística ordinal usando a PNAD 2008. Ciência \& Saúde Coletiva, 16(9), 3769-3780. https://doi.org/10.1590/S1413-81232011001000013

Morris, A. J., \& Burke, F. J. (2001). Primary and secondary dental care: how ideal is the interface?. British dental journal, 191(12), 666-670. https://doi.org/10.1038/sj.bdj.4801263

Nuttall, N. M., Steed, M. S., \& Donachie, M. A. (2002). Referral for secondary restorative dental care in rural and urban areas of Scotland: findings from the Highlands Et Islands Teledentistry Project. British Dental Journal, 192(4), 224-228. https://doi.org/10.1038/sj.bdj.4801339

Oliveira, A. P. C., Gabriel, M., Poz, M. R. D., \& Dussault, G. (2017). Desafios para assegurar a disponibilidade e acessibilidade à assistência médica no Sistema Único de Saúde. Ciência \& Saúde Coletiva, 22(4), 1165-1180. https://doi.org/10.1590/1413-81232017224.31382016

Pereira A.S. et al. (2018). Metodologia da pesquisa científica. [e-book]. Santa Maria. Ed. UAB/NTE/UFSM. Disponível em: https://repositorio.ufsm.br/bitstream/handle/1/15824/Lic_Computacao_Metodologia-Pesquisa-Cientifica.pdf?sequence=1 .

Porto, S. M., Ugá, M. A., \& Moreira, R. S. (2011). Uma análise da utilização de serviços de saúde por sistema de financiamento: Brasil 1998-2008. Ciência \& Saúde Coletiva; 16(9), 3795-3806. https://doi.org/10.1590/S1413-81232011001000015

Scott, G., Brodeur, J. M., Olivier, M., \& Benigeri, M. (2002). Parental factors associated with regular use of dental services by second-year secondary school students in Quebec. Journal Canadian Dental Association, 68(10), 604-608.

Teusner, D. N., Brennan, D. S., \& Spencer, A. J. (2015). Associations between level of private dental insurance cover and favourable dental visiting by household income. Australian Dental Journal, 60(4), 479-489. https://doi.org/10.1111/adj.12268

Traverso-Yépez, M., \& Morais, N. A. (2004). Reivindicando a subjetividade dos usuários da Rede Básica de Saúde: para uma humanização fazer atendimento. Cadernos de Saúde Pública, 20(1):80-88. https://doi.org/10.1590/S0102-311X2004000100022

Vieira Junior, W., \& Martins, M. (2015). Idosos e planos de saúde no Brasil: análise das reclamações recebidas pela Agência Nacional de Saúde Suplementar. Ciência \& Saúde Coletiva, 20(12), 3817-3826. https://doi.org/10.1590/1413-812320152012.11082014

Yamamoto, T. S., Machado, M. T. C., \& Silva Junior, A. G. (2015). Educação permanente em saúde como prática avaliativa amistosa à integralidade em Teresópolis, Rio de Janeiro. Trabalho, Educação e Saúde, 13(3), 617-637. https://doi.org/10.1590/1981-7746-sip00058 
Research, Society and Development, v. 10, n. 6, e56110615888, 2021

(CC BY 4.0) | ISSN 2525-3409 | DOI: http://dx.doi.org/10.33448/rsd-v10i6.15888

Zanetti, C. H. G. (1999). A crise da odontologia brasileira: as mudanças estruturais do mercado de serviços e o esgotamento do modo de regulação curativo de massa. Revista Ação Coletiva, 2(3), 11-24.

Ziroldo, R. R., Gimenes, R. O., \& Castelo Jr, C. (2013). A importância da saúde suplementar na demanda da prestação dos serviços assistenciais no Brasil. Mundo Saúde, 37(2), 216-221. 